\title{
Mind the gap: toward an integrative science of the brain and crime
}

\author{
Eyal Aharoni ${ }^{1}$ - Nathaniel E. Anderson ${ }^{2}$ - J. C. Barnes ${ }^{3} \cdot$ Corey H. Allen $^{4}$. \\ Kent A. Kiehl ${ }^{2}$
}

Published online: 16 August 2019

(C) Springer Nature Limited 2019

In the recent article, Criminalizing the brain: Neurocriminology and the production of strategic ignorance, Fallin et al. (2018) argue that most neuroscientists working with antisocial populations are guilty of suppressing, obfuscating, and erasing legitimate social explanations for criminal behavior as part of a strategic attempt to tout their reductionist preconceptions of behavior and to advance their own professional, extra-scientific agendas. While we applaud their call for greater inclusion of social/ contextual factors into neurocriminological research, we find that they overstate the case against neurocriminology and understate important efforts by this emerging community to generate significant and cross-disciplinary contributions to the understanding of antisocial behavior. Learning to identify and avoid such mischaracterizations is critical for the pursuit of much-needed interdisciplinary research and collaboration on the prediction, explanation, and remediation of antisocial behavior. By critically evaluating Fallin et al.'s claims, we hope to strike a conciliatory balance, which is more likely to promote integration rather than antagonism between disciplines.

Fallin and colleagues correctly describe biosocial criminology as an emerging discipline that integrates historically neglected biological factors and neuroscientific methods in the study of antisocial behavior. Indeed, this paradigm has demonstrated the value of genetics (Brunner et al. 1993), physiology (Latvala et al. 2015), biochemistry (Coccaro et al. 1998), and neuroimaging (Anderson and Kiehl 2012) for

Eyal Aharoni

eaharoni@gsu.edu

1 Department of Psychology, Georgia State University, P.O. Box 5010, Atlanta, GA 30302-5010, USA

2 Mind Research Network, 1101 Yale Blvd NE, Albuquerque, NM 87106-4188, USA

3 School of Criminal Justice, University of Cincinnati, Cincinnati, OH 45221-0389, USA

4 Neuroscience Institute, Georgia State University, P.O. Box 5030, Atlanta, GA 30302-5030, USA 
understanding individual differences that promote behaviors that are at odds with the law. Neurocriminology specifically focuses on properties of the brain and nervous system that guide our behavior. This stands out as a particularly useful platform for studying biological influences on crime as the brain is the most proximal biological antecedent to behavior on which many other influences converge. Genetics, social factors, environmental stressors, disease, and injury all exert measurable, cumulative influences on the brain and behavior. We therefore suggest that Fallin and colleagues go too far when they characterize neurocriminology as a corrective force pitted against traditional sociological theories. A goal of any scientific agenda should be to expand and improve on useful models and replace or adapt intractable ones. However, there is nothing inherent in a neurocriminological agenda that is antagonistic to well-established sociological theory.

Fallin et al. argue that social variables are being ignored by neuroscientists studying antisocial behavior. To their credit, there are innumerable examples of social and contextual factors that influence one's risk of antisocial behavior. One obvious example is socioeconomic status (Barkan and Rocque 2018). But there are at least as many examples of contemporary neuroscience embracing the position that socioeconomic and biological domains are interrelated. For instance, many studies have shown that low socioeconomic status may negatively affect neurocognitive function (e.g., Hackman et al. 2010; Kishiyama et al. 2009; McDermott et al. 2019). These scientists recognize that there is no rivalry between known social factors and biological factors since much variation in the brain is experience-dependent, and combinations of these variables inevitably shape criminal offense propensity (e.g., Beaver 2017; Caspi et al. 2002; Pardini et al. 2014; Viding et al. 2005).

Fallin et al. also assert that neurocriminologists have not merely neglected social factors, but have actively "suppressed," "obfuscated," and "erased" them as part of a strategic attempt to advance a reductionist worldview and to jockey for professional status. To support these claims, the authors criticize the variable selection, ecological validity, and interpretation of a handful of studies. Here we review some problems with this argument.

First, this argument confuses biological reductionism with the necessary practice of inductive empirical research. All models are 'reductive' in the practical sense that they cannot measure everything, and so they seek to test explicit hypotheses using a limited number of variables, operationalized in controlled contexts. In other words, “... all models are wrong, but some are useful" (Box and Draper 1987). Evidence that neuroscientists have made measurement compromises does not imply that they are touting biological reductionism. Fallin et al. imply that it is the responsibility of neurocriminologists to develop exhaustive theories of criminal behavior, but this would be an unrealistic expectation for any discipline. A choice to target biological factors could be based on the reasonable and non-reductionist view that biological and social descriptions comprise different levels of analysis, and thus offer unique but complementary perspectives on human behavior.

Second, Fallin et al. assert that neurocriminologists are peddling a deterministic and nativistic view, and they assume that neurobiological factors are rigid and innate while social factors are dynamic and acquired exclusively from environments (p. 17). These assumptions are at odds with the mainstream contemporary 
biopsychosocial framework. Under this framework, human social behavior results from dynamic, bidirectional interactions between the brain and social environments. Language development, face perception, and contagious yawning are just a few examples that follow this pattern (Simion and Giorgio 2015; Teive et al. 2018; Tierney and Nelson 2009).

Third, Fallin et al. describe the "thinness" of experimental tasks as a way to criticize the works' ecological validity. It is true that many neurocognitive tasks use impoverished stimuli, but this is often for the justifiable reason of experimental control. This strategy ensures that any effects can be clearly attributed to the planned manipulations. But there is an inherent tradeoff between experimental control and ecological validity. The investigator carries discretion to determine how to balance this tradeoff depending on the nature of her research question and goals. In the case of a young and highly multivariate field such as neurocriminology, it seems justified to prioritize high experimental control as a way to constrain the problem before introducing more complex representations of a given phenomenon, even though it may limit generalizability.

Methodologically, the qualitative strategy employed to justify Fallin et al.'s conclusions strongly limits some of the inferences made. First, their article made no mention of rater blinding, inter-rater reliability, or publication of their coding taxonomy or data, which are known best practices in research. Second, it did not cite a majority of the articles included in their analysis, leaving readers unable to validate their claims. Third, instead of taking a random sample of the neurocriminology literature, their search parameters selectively targeted medicalized disorders that are, to a large extent, characterized biologically. This decision likely increased the proportion of search results that employed a biological level of analysis, thus stacking the cards in favor of the authors' hypothesis.

Despite this selection bias, the majority of studies cited did in fact include socially relevant variables in their statistical models. For example, Aharoni et al. (2013) included a measure of drug and alcohol abuse in their risk assessment models because of its well-established ability to predict criminal behavior and recidivism (Dowden and Brown 2002). They then tested the incremental predictive utility of a functional brain metric beyond these other socially relevant variables. Fallin et al. suggested that by controlling for drug and alcohol abuse, Aharoni et al. were hiding the fact that such factors might provide an alternative (i.e., social) explanation for their results. To the contrary, partitioning the variance attributed to biological and behavioral factors enabled Aharoni et al. to explicitly estimate their independent effects.

Fallin et al. also infer that, because Aharoni et al. collected race demographics but did not include them in their risk assessment models, it must be that they deny the relevance of racially discriminatory criminal justice practices. This surprised us because reporting descriptive statistics on sample demographics is a common convention in neuroscience research-enabling readers to replicate the work, or at least understand its limitations. More importantly, it is often unjustified to include measures like race in risk models. Scientifically, researchers must consider limitations in variance and statistical power, or should have a compelling theoretical reason for expecting that a given variable, such as a person's race, 
directly causes crime (which we absolutely do not). Researchers could be tempted to use race as a proxy for another factor, such as ancestry. Ancestry itself could be of interest in certain kinds of analyses, for instance, in genetics analysis, where creating more homogeneity in common variation between subjects improves power for detecting individual variation related to other traits of interest. But race is known to be a poor proxy for ancestry because the construct of race is, by and large, socially constructed (Duster 2015; Sen and Wasow 2016). Yet, unlike other social factors like economic disadvantage, there are no viable theories that race causes crime. As such, any associations observed between race and crime likely reflect a perceiver bias. This may help explain why the use of race as an indicator in risk assessment instruments is widely recognized as ethically problematic (and, in many cases, illegal [e.g., Adarand Constructors Inc. v. Peña 1995; Gonzales v. Quarterman 2006]): it carries grave potential to stigmatize defendants and to mislead fact finders into thinking that race itself somehow causes crime, producing a more biased, self-perpetuating cycle. Thus, if race information is included in criminological models, it should be used, not as a means to test its direct effects, but potentially to partial out its interactive effects (Barnes 2018).

A larger concern about Fallin et al.'s rhetorical strategy is the imposition of a double bind. If well-intentioned scientists do not include conventionally social factors in their models, Fallin and colleagues accuse them of being biological reductionists. If the investigators control for such factors, the authors accuse them of trivializing it. And if these neurocriminologists include social factors, they risk being blamed of perpetuating harmful social biases should the results cast social groups in a negative light. A constructive dialog between sociological and neuroscientific fields should respect common standards of falsifiability.

Fallin et al. do consider that the motivations of neurocriminologists might be innocent, but this placation is overshadowed by strong language decrying the "production" of ignorance and the "suppression" and "erasure" of the so-called inconvenient facts. In the use of such active voicing, the authors imply a sin of commission, but at worst, their evidence reveals justifiable research design decisions. To be consistent, their hyperbolic logic must apply equally to sociological research that "suppresses" biological theories simply because it does not explicitly test them. Such logic would be hardly fair to sociologists.

While sociologists and neuroscientists alike may both be vulnerable to limitations in experimental designs, or imperfect interpretations of data, there is generally more evidence that scientists in both camps are principally motivated to improve and increase knowledge rather than to obscure it. Further, when imperfections and limitations arise, we see evidence that these are acknowledged in good faith, rather than disguised in malice or conceit. To suggest otherwise seems only to undermine efforts to diversify and integrate the disciplines. We hope, at the least, we have clarified that neuroscience need not be viewed as a competing force seeking to replace other disciplines, but rather as one of the several lenses through which to examine complex behavior. Understanding criminal behavior is, of course, a very complex problem. So, its careful study could benefit greatly from more constructive dialog and collaboration between sociological and neuroscientific disciplines. 


\section{References}

Adarand Constructors, Inc. v. Peña, 515 U.S. 200 (1995).

Aharoni, E., G.M. Vincent, C.L. Harenski, V.D. Calhoun, W. Sinnott-Armstrong, M.S. Gazzaniga, and K.A. Kiehl. 2013. Neuroprediction of Future Rearrest. Proceedings of the National Academy of Sciences United States of America 110: 6223-6228.

Anderson, N.E., and K.A. Kiehl. 2012. The Psychopath Magnetized: Insights From Brain Imaging. Trends in cognitive sciences 16 (1): 52-60.

Barkan, S.E., and M. Rocque. 2018. Socioeconomic Status and Racism as Fundamental Causes of Street Criminality. Critical Criminology 26 (2): 211-231.

Barnes, J.C. 2018. A Constructivist View of Race in Modern Criminology. Journal of Criminal Justice 59: 81-86.

Beaver, K. 2017. Biosocial Theories of Crime. London: Routledge.

Box, G.E., and N.R. Draper. 1987. Empirical Model-Building and Response Surfaces. New York: John Wiley.

Brunner, H.G., M. Nelen, X.O. Breakefield, H.H. Ropers, and B.A. Van Oost. 1993. Abnormal Behavior Associated with a Point Mutation in the Structural Gene for Monoamine Oxidase A. Science 262 (5133): 578-580.

Caspi, A., J. McClay, T.E. Moffitt, J. Mill, J. Martin, I.W. Craig, A. Taylor, and R. Poulton. 2002. Role of Genotype in the Cycle of Violence in Maltreated Children. Science 297 (5582): 851-854.

Coccaro, E.F., R.J. Kavoussi, R.L. Hauger, T.B. Cooper, and C.F. Ferris. 1998. Cerebrospinal Fluid Vasopressin Levels: Correlates with Aggression and Serotonin Function in Personality-Disordered Subjects. Archives of General Psychiatry 55 (8): 708-714.

Dowden, C., and S.L. Brown. 2002. The Role of Substance Abuse Factors in Predicting Recidivism: A Meta-analysis. Psychology, Crime and Law 8 (3): 243-264.

Duster, T. 2015. A post-genomic surprise The molecular reinscription of race in science, law and medicine. The British Journal of Sociology 66 (1): 1-27.

Fallin, M., O. Whooley, and K.K. Barker. 2018. Criminalizing the brain: Neurocriminology and the production of strategic ignorance. BioSocieties 1: 25. https://doi.org/10.1057/s41292-018-0135-y.

Gonzales v. Quarterman, 458 F.3d 384 (2006).

Hackman, D.A., M.J. Farah, and M.J. Meaney. 2010. Socioeconomic Status and the Brain: Mechanistic Insights from Human and Animal Research. Nature Reviews Neuroscience 11 (9): 651.

Kishiyama, M.M., W.T. Boyce, A.M. Jimenez, L.M. Perry, and R.T. Knight. 2009. Socioeconomic Disparities Affect Prefrontal Function in Children. Journal of Cognitive Neuroscience 21 (6): 1106-1115.

Latvala, A., R. Kuja-Halkola, C. Almqvist, H. Larsson, and P. Lichtenstein. 2015. A Longitudinal Study of Resting Heart Rate and Violent Criminality in More Than 700000 Men. JAMA Psychiatry 72 (10): 971-978.

McDermott, C.L., J. Seidlitz, A. Nadig, S. Liu, L.S. Clasen, J.D. Blumenthal, P.K. Reardon, F. Lalonde, D. Greenstein, R. Patel, and M.M. Chakravarty. 2019. Longitudinally Mapping Childhood Socioeconomic Status Associations with Cortical and Subcortical Morphology. Journal of Neuroscience 39 (8): 1365-1373.

Pardini, D.A., A. Raine, K. Erickson, and R. Loeber. 2014. Lower Amygdala Volume in Men is Associated with Childhood Aggression, Early Psychopathic Traits, and Future Violence. Biological Psychiatry 75 (1): 73-80.

Sen, M., and O. Wasow. 2016. Race as a Bundle of Sticks: Designs That Estimate Effects of Seemingly Immutable Characteristics. Annual Review of Political Science 19: 499-522.

Simion, F., and E.D. Giorgio. 2015. Face Perception and Processing in Early Infancy: Inborn Predispositions and Developmental Changes. Frontiers in Psychology 6: 969.

Teive, H.A., R.P. Munhoz, C.H.F. Camargo, and O. Walusinski. 2018. Yawning in Neurology: A Review. Arquivos de Neuro-Psiquiatria 76 (7): 473-480.

Tierney, A.L., and C.A. Nelson III. 2009. Brain Development and the Role of Experience in the Early Years. Zero to Three 30 (2): 9.

Viding, E., R.J.R. Blair, T.E. Moffitt, and R. Plomin. 2005. Evidence for Substantial Genetic Risk for Psychopathy in 7-Year-Olds. Journal of Child Psychology and Psychiatry 46 (6): 592-597.

Publisher's Note Springer Nature remains neutral with regard to jurisdictional claims in published maps and institutional affiliations. 
Eyal Aharoni is an Assistant Professor of Psychology and Philosophy at Georgia State University. His research investigates criminal, moral, and legal decision making and the role of neurocognitive predictors of antisocial behavior. This work is funded by the National Science Foundation (Grant 1829495).

Nathaniel E. Anderson is an Assistant Professor of Translational Neuroscience at The Mind Research Network in Albuquerque, NM. His research focuses on variations in social, biological, and neurocognitive systems that promote antisocial behavior and maladaptive decision-making.

J. C. Barnes is an associate professor in the School of Criminal Justice at the University of Cincinnati. His research seeks to identify how genetic and environmental factors combine to impact criminological phenomena. Recent works can be found in outlets such as Behavior Genetics, Criminology, and Psychological Science.

Corey H. Allen is a Ph.D. Candidate in Neuroscience, with a concentration in Neuroethics, at Georgia State University. His research investigates moral decision making, the use of neurocognitive markers for the prediction of antisocial behavior, and the ethical implications of such use.

Kent A. Kiehl is a Professor of Psychology, Neuroscience and Law at the University of New Mexico and Executive Science Officer of the non-profit Mind Research Network in Albuquerque, NM. Dr. Kiehl conducts clinical neuroscience research of major mental illnesses, with special focus on criminal psychopathy, substance abuse, and psychotic disorders. 